\title{
Urachal Abscess Can Be Confused With Pelvic Appendicitis
}

\author{
Chi D. $\mathrm{Ha}^{*}$
}

Department of Surgery, Pinnacle Health Hospitals, Harrisburg, PA 17104, USA

\begin{abstract}
Urachal remnant is a rare disorder of the genitourinary system. It frequently manifests with urine leakage from the umbilicus but can sometimes present as an infected process. We herein describe a case of a six-year-old male presenting with an urachal abscess initially thought to be a ruptured pelvic appendicitis.
\end{abstract}

\section{CASE REPORT}

A 6-year-old male with intermittent dysuria over a tenweek period presented with a low-grade fever, moderate suprapubic pain, and worsening dysuria for the last seven days. The patient had moderate tenderness and firmness over the suprapubic area and a normal genitourinary system exam. There was no sign of a periumbilical drainage or infection. The laboratory studies showed a white blood cell count of $23.4 \times 10^{9}$ cells/L without a left shift, and a C-reactive protein level of $14.8 \mathrm{mg} / \mathrm{L}$. The urinary analysis and culture failed to show an urinary tract infection. A computed tomographic scan revealed a necrotic mass on the right lateral aspect of the urinary bladder (Fig. 1). The most likely diagnosis at this time was ruptured pelvic appendicitis, but an urachal abscess was also in the differential diagnosis; therefore, we decided to surgically explore the extra-peritoneal space first for the possibility of encountering an urachal abscess.

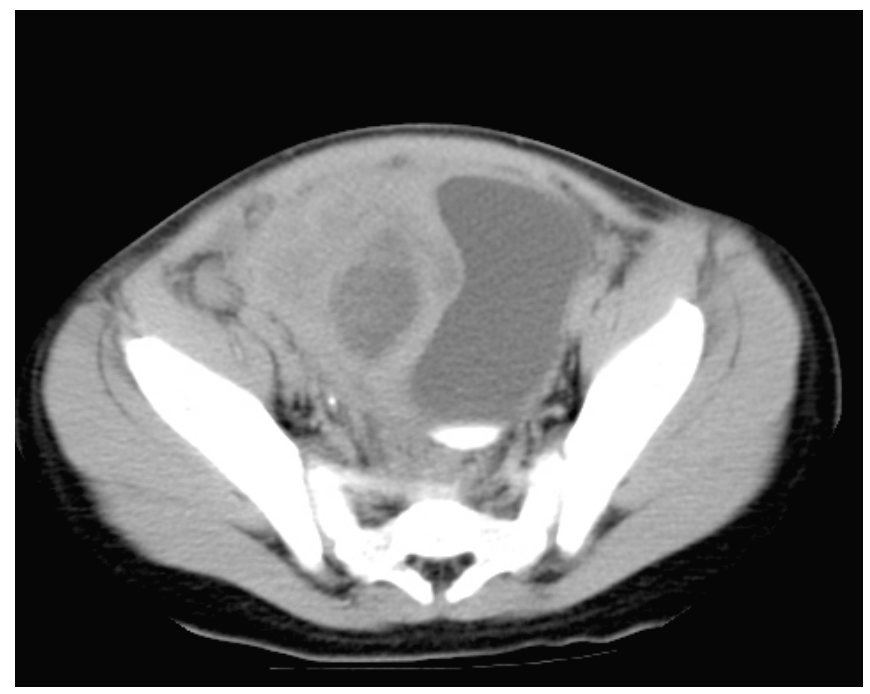

Fig. (1). A computed tomographic image showing a $2.3 \times 3.9-\mathrm{cm}$ necrotic mass on the right lateral aspect of the urinary bladder.

An extraperitoneal exploration was attempted through a transverse five-centimeter incision right lateral to the rectus abdominis at the level of the anterior superior iliac spine. Due to the inflammation and fragility of the anterior

*Address correspondence to this author at the Pinnacle Health Hospitals, 205 S. Front St., BMAB-9, Harrisburg, PA 17104, USA; Tel: (717) 2318755; Fax: (717) 231-8756; E-mail: ha_md@surgeonsknot.com abdominal wall tissue, the peritoneum was incidentally violated, and a large abscess on the right lateral aspect of the urinary bladder was immediately encountered. This purulent collection ruptured during an attempt to bluntly isolate it from the adhered small bowel loops, cecum, and omentum. An elongated appendix was found with its distal portion included in the decompressed necrotic mass. This intraoperative finding favored the diagnosis of a ruptured appendicitis. As a result, an appendectomy was performed by clamping and transecting the appendix and the mesoappendix at their connections to the cecum. The contiguous inflammatory tissue at the distal appendix was removed together with the appendix (Fig. 2) followed by a copious irrigation of the intra-abdominal cavity. A closed suction drain was placed. Surprisingly, the microscopic study indicated a benign and intact appendix adhering distally to a mass of necrotic tissue. No intact urachal epithelium was found within the removed portion of the necrotic mass.

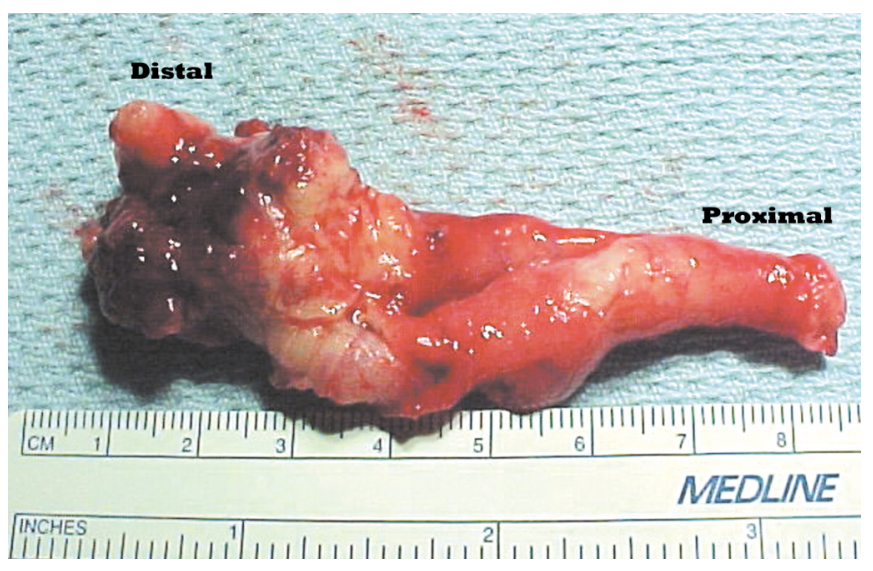

Fig. (2). The removed appendix with its distal portion adhered to the abscess.

The patient's postoperative course was complicated by a urinary leak from the right lateral aspect of the bladder confirmed by a cystogram (Fig. 3). The leak spontaneously resolved after ten days of Foley catheter decompression of the bladder and closed suction drainage of the pelvic cavity. The patient was symptom-free at his four- and eight-week follow-ups.

\section{DISCUSSION}

The urachus is an embryonic connection between the allantois and the cloaca. During the fourth to seventh week of gestation, the urorectal septum grows caudally and divides the cloaca into the urogenital sinus anteriorly and anal canal 
posteriorly. The uppermost part of the urogenital sinus develops into the urinary bladder whose dome connects to the allantois by the epithelialized urachus. The urachus elongates as the bladder descends, and eventually obliterates by the fifth to the seventh month of gestation, leaving behind a three to ten centimeter fibrous remnant (the median umbilical ligament) extending from the bladder dome to the base of the umbilicus [1,2]. The urachus may merge with one or both remnants of the umbilical arteries (the medial umbilical ligaments) and becomes deviated either left or right.

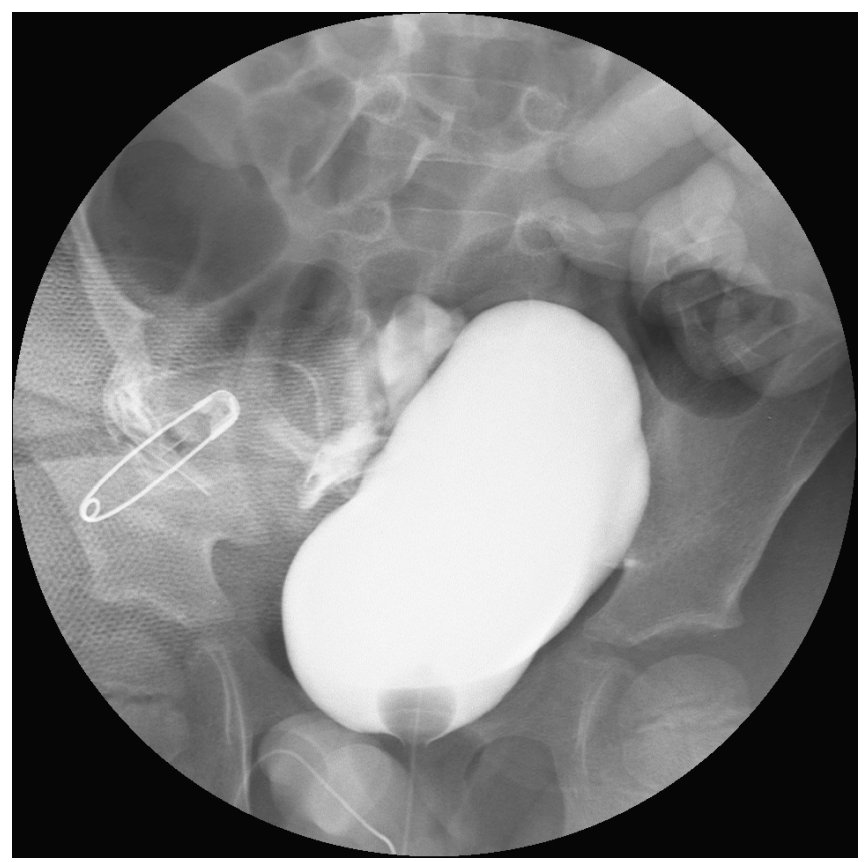

Fig. (3). A cystogram on the $6^{\text {th }}$ post-operative day showing a mass effect with the leakage of contrast on the right lateral aspect of the bladder.

Urachal anomalies are rare disorders of the genitourinary tract and have a 5:1 male:female predisposition. The incidence remains unclear. Nix et al. reports three cases in 200,000 admissions in Boston and three cases in 1,168,760 admissions in New York [3]. Meanwhile, Blichert-Toft et al. reports only five cases in 40,000 surgical admissions [1]. Urachal anomalies can be divided into five categories [4]. Congenital patent urachus accounts for $50 \%$ of all anomalies and results from a failure of descending of the urinary bladder [1,2]. An intact, epithelialized connection between the bladder and the umbilicus is expected. These patients usually present during infancy with a clear umbilical drainage which can be confused with the drainage from a vitelline duct remnant. The next most common category is urachal cyst which accounts for $30 \%$ of the cases and results from an incomplete obliteration of the urachus. Although most of these epithelialized cysts are asymptomatic, patients can present anytime from infancy to adolescence with either an acute or chronic periumbilical infection or abscess [1]. The next two categories, non-epithelialized urachal sinuses and alternating urachal sinuses, together account for approximately $15 \%$ of the cases, and can present with either periumbilical drainage or infection. The last category, urachal diverticulum at the dome of a bladder, is rare and usually found incidentally.

The most common clinical presentation of urachal anomalies is umbilical drainage and/or periumbilical in- flammation [4]. Umbilical drainage is in general a presentation of a patent urachus or urachal sinus, and is mostly detected during the first four weeks of life. Periumbilical inflammation, however, is a presentation of an infected urachal cyst or sinus and can present later on in life. Most patients with infected urachal remnants do not have a systemic or laboratory evidence of an infection such as fever or leukocystosis $[4,5] .80 \%$ of isolated bacteria from the infected urachal fluids are staphylococcal species, which suggests the origin of the infection from the skin rather than from the genitourinary tract $[4,6]$. Although dysuria is a common symptom, a urinary analysis and culture serve as a poor indicator of urachal anomalies, infected or not [4]. The broad differential diagnosis of an urachal infection includes appendicitis, Meckel or colonic diverticulitis, pelvic inflammatory disease, perforated viscus, acute prostatitis, urinary tract infection, cystitis, pyelonephritis, and strangulated umbilical, inguinal, or femoral hernia. Of these, only the first four would potentially cause an abscess formation. Associated anomalies are rare and include meatal stenosis, hypospadias, umbilical hernia, cryptorchism, anal atresia, omphalocele, crossed renal ectopia, ureteropelvic obstruction, and most frequently, vesicoureteral reflux $[4,6,7]$. Malignant degenerations of urachal remnants, usually found in men from $40-70$ years of age, are also rare and account for less than $0.5 \%$ of all bladder cancers [8,9], and therefore, do not warrant a prophylactic screening and excision. The most common malignant cell type is adenocarcinoma ( $90 \%$ of all cases), followed by sarcoma, transitional cell carcinoma, and squamous cell carcinoma [9].

The diagnosis of urachal anomalies is mainly clinical $[5,6,7]$. An ultrasonography is the confirmatory test of choice due to its sensitivity and specificity of $90-100 \%$ $[4,6,7]$. The common ultrasonic finding is a thickened tubular structure along the midline below the umbilicus tracking to the bladder dome. A computed tomography scan may also reveal the extent of abdominal wall involvement and the possibility of intra-abdominal abscess; however, radiation exposure in children should be avoided if possible. A voiding cystourethrogram and a cystoscopy do not usually provide additional information $[4,5,10]$. A sinogram can be performed in patients with periumbilical drainage to aid in the differentiation between vitelline duct and urachal remnants $[4,10]$.

Surgical excisions of the remnants are the definitive treatment for urachal anomalies. For an uncomplicated case, a one-stage extraperitoneal approach is used to remove the urachal remnants together with a small cuff of the bladder, followed by a two-layer closure of the bladder defect $[1,2,4$ $6]$. This can be done laparoscopically [10]. For complicated cases, especially with abscess, the traditional two-stage approach is used to limit the amount of bladder resection and to reduce the risk of injury of the adjacent intraperitoneal structures [1,2,4-7]. Stage one involves an incision and drainage, and a use of broad-spectrum antibiotics to control the infection [4]. Stage two involves an elective excision of the urachal remnant. If not excised, there is a $30 \%$ risk of recurrence of the infection and a small possibility of a malignant degeneration $[1,3]$. It has been shown that the two-stage approach results in fewer complications such as wound infection or urinary bladder leak as compared to the one-stage approach, even in the age of advanced antibiotics [4]. 
In our case, although there is no periumbilical infection or drainage on the examination, the pelvic abscess was tightly associated with the anterior abdominal wall and the right lateral aspect of the bladder. The urachal remnant might have fused with the right medial ligament and been developing into a large abscess over the ten-week period. The adhered pelvic appendix made the differentiation between an urachal abscess and appendicitis difficult on computed tomography scan and during surgery. However, the appendix was wholly benign on the microscopic examination. We hypothesize that a necrotic and ruptured urachal cyst was "walled off" within the pelvis on the right lateral aspect of the urinary bladder.

\section{CONCLUSION}

An urachal abscess, although a rare disease, should be considered in the differential diagnosis of a pelvic abscess, especially in children. Besides a thorough history and physical exam, ultrasonography is the diagnostic modality of choice, but computed tomography can add some information on the extent the infected process and help in differentiating this disease from the broad list of similar diseases. The treatment of choice for a complicated urachal abscess involves a two-staged surgical approach together with the use of broad-spectrum antibiotics.

\section{ACKNOWLEDGEMENT}

The author owes his deepest gratitude to Dr. Domingo Alvear, Chief of Pediatric Surgery at Harrisburg Hospitals, who encourages the author to publish this article.

\section{REFERENCES}

[1] Blichert-Toft M, Nielsen OV. Congenital patent urachus and acquired variants. Diagnosis and treatment. Review of the literature and report of five cases. Acta Chir Scand 1971; 137: 807-14.

[2] Gearhart JP, Jeffs RD. Exstrophy-epispadias complex and bladder anomalies. In: Walsh PC, Retik AB, \& Vaughn ED, Campbell's Urology. Vol 2. Ed 7. Philadelphia: Saunders; 1998: 1985-1987.

[3] Nix JT, Menville JG, Albert M, et al. Congenital patent urachus. J Urol 1958; 79: 264-73.

[4] McCollum MO, MacNeily AE, Blair GK. Surgical implications of urachal remnants: presentation and management. J Pediatr Surg 2003; 38: 798-803.

[5] MacNeily AE, Koleilat N, Kiruluta HG, et al. Urachal abscesses: protean manifestations, their recognition, and management. Urology 1992; 40: 530-5.

[6] Suita S, Nagasaki A. Urachal remnants. Seminars in Pediatric Surgery 1996; 5: 107-115.

[7] Rich RH, Hardy BE, Filler RM. Surgery for anomalies of the urachus. J Pediatr Surg 1983; 18: 370-2.

[8] Henly DR, Farrow GM, Zincke H. Urachal cancer: role of conservative surgery. Urology 1993; 42: 635-9.

[9] Sheldon CA, Clayman RV, Gonzales R, et al. Malignant urachal lesions. J Urol 1984; 131: 1-8.

[10] Cilento BG Jr, Bauer SB, Retik AB, et al. Urachal anomalies: defining the best diagnostic modality. Urology 1998; 52: 120-2. 